\title{
NUEVAS TERAPIAS SISTÉMICAS PARA EL TRATAMIENTO DEL MELANOMA*
}

\author{
Drs. Nicolás Droppelmann M. ${ }^{1,2}$, Augusto León R..$^{1,2}$, Ignacio Goñi E. ${ }^{1,2}$, \\ Hernán González D. ${ }^{1,2}$, Francisco Domínguez C. ${ }^{1,2}$, Mauricio Camus A. ${ }^{1,2}$, \\ Bruno Nervi N. ${ }^{1,3}$, Pablo Uribe G. ${ }^{1,4}$, Montserrat Molgó N.., 4 , Francisco Acevedo C. ${ }^{1,3}$
}

1 Unidad de Melanoma y Cáncer de Piel.

2 Departamento de Cirugía Oncológica y Maxilofacial.

3 Departamento de Hemato Oncología.

4 Departamento de Dermatología.

División de Cirugía, Pontificia Universidad Católica de Chile.

Santiago, Chile.

\section{New systemic therapies for melanoma}

Melanoma represents a disease with high tendency to metastasize locally, regionally to the lymph nodes, and systemically to lungs, liver, brain and bone. The surgical treatment has been the cornerstone to treat melanoma patients, with excellent results in early stage, but with poor effectiveness in advance disease with systemic metastases. Traditional chemotherapy with cytotoxic agents has shown little impact on stage IV patients, which has motivated new investigation and innovation in this field. During last 4 years, FDA has approved new therapies for the treatment of metastatic or unresectable melanoma, mostly based in immunotherapy and signal transduction inhibitors, with very promising results. The surgeons treating these patients must be updated about these new therapies, which represent a new treatment opportunity for our patients.

Key words: Melanoma, metastatic, immunotherapy, chemotherapy, treatment, new advances.

\section{Resumen}

El melanoma es un tumor maligno que tiene una alta tendencia a metastizar localmente, regionalmente al sistema linfático, y en forma sistémica, preferentemente a pulmones, hígado, cerebro y hueso. La cirugía es el principal tratamiento para el melanoma, con excelentes resultados en estadios precoces, pero escasa utilidad en etapas avanzadas con enfermedad sistémica. Tradicionalmente los tratamientos en base a quimioterapias citotóxicas han aportado poco beneficio a los pacientes en etapa IV, lo que ha motivado una amplia investigación e innovación en esta área. Actualmente la Food and Drug Administration (FDA) ha aprobado nuevas terapias para el tratamiento del melanoma metastásico, irresecable o recurrente, en base a drogas

\footnotetext{
Los autores declaran no tener conflicto de interés.

Correspondencia: Dr. Nicolás Droppelmann M. ndroppel@med.puc.cl
}

*Recibido el 13 de abril de 2015 y aceptado para publicación el 5 de mayo de 2015. 
inmunomoduladoras y drogas que bloquean la transducción de señales, con resultados muy promisorios. Los cirujanos que tratamos pacientes con melanoma, debemos estar al día sobre el uso de estas nuevas terapias que representan una nueva oportunidad de tratamiento para nuestros pacientes.

Palabras clave: Melanoma, metastásico, inmunomodulación, quimioterapia, tratamiento.

\section{Introducción}

El melanoma representa un problema de salud importante en Chile y el mundo. Para hacernos una idea más clara de este problema, debemos mirar las cifras de Estados Unidos, a la espera de poder generar estadísticas con respecto a este tema en Chile.

En el 2015, se estima que se van a diagnosticar 73.870 nuevos casos de melanoma invasor en Estados Unidos, 9.940 personas van a morir por esta causa ${ }^{1}$.

La única forma de curar un paciente con melanoma es a través de la cirugía. La resección correcta del primario con márgenes adecuados y el correcto manejo de linfonodos regionales (biopsia de linfonodo centinela y disección linfática cuando esté indicado) son puntos críticos para alcanzar este resultado. Además, la resección de metástasis en pacientes etapa IV también ha demostrado ser un tratamiento eficaz para un grupo de pacientes seleccionados.

Sin embargo, aquellos pacientes en etapa III considerados irresecables, pacientes en etapa IV, y aquellos con melanoma recurrente, se escapan del beneficio que el bisturí puede aportar.

Hace tan sólo cinco años, a este grupo de pacientes, sólo podíamos ofrecerle algunos tipos de quimioterapia y/o interleukina 2 (IL2). Lamentablemente, muy pocos pacientes se beneficiaban con este tipo de tratamiento, aunque por algunas razones que no conocemos bien, algunos de ellos (muy pocos) mostraban una mejoría considerable.

Hoy día en cambio, nuevas terapias han demostrado avances que anteriormente no se habían logrado en términos de prolongación de la sobrevida de estos pacientes.

El mayor estudio y entendimiento del sistema inmunológico, ha llevado al desarrollo de nuevas terapias inmunomoduladoras como el ipilimumab, un anticuerpo monoclonal que se une a CTLA-4, bloqueando su habilidad de inhibir la activación, proliferación y función de las células $\mathrm{T}$, que ha probado mejorar la sobrevida en estudios fase III en melanoma metastásico ${ }^{2,3}$. En la misma línea, se han desarrollado anticuerpos anti PD1 (Programmed death 1), recientemente destacados por ASCO como uno de los avances más importantes del año en cáncer ${ }^{4,5}$.

Se ha avanzado también durante estos cuatro años, en la identificación de mutaciones del gen $\mathrm{BRAF}$ en la vía MAPK (mitogen-activated protein kinase), que ocurre en al menos un 50\% de los pacientes con melanoma cutáneo, y que puede ser tratado con terapias blanco (terapias target) con pequeñas moléculas que inhiben la proteína $\mathrm{BRAF}$, MEK o ambas 6 .

De esta forma, estamos en presencia de dos nuevas vías que, si bien aún han demostrado resultados modestos en términos de sobrevida, han sido por lejos los avances más grandes que hasta ahora se habían tenido en melanoma, y mejor aun, nos muestran un muy buen camino para continuar el desarrollo de terapias sistémicas para esta enfermedad.

Es por esto, que los cirujanos debemos considerar a nuestros pacientes metastásicos, irresecables o recurrentes para recibir, idealmente en un ensayo clínico, estas nuevas terapias aprobadas por la FDA.

\section{Nuevas drogas}

\section{Inmunoterapias}

\section{Anti-CTLA-4: Ipilimumab}

Del punto de vista inmunológico, cuando un antígeno de melanoma (por ejemplo gp100) es procesado por una célula presentadora de antígeno (CPA), este antígeno es exhibido en su superficie a través de un receptor transmembrana (MHC-II) siendo reconocido por el linfocito. Este linfocito $\mathrm{T}$ ahora activado, prolifera, madura y circula en la periferia donde eventualmente se encontrará con el antígeno que le fue presentado, para producir su efecto citotóxico.

Existen "checkpoints" inmunológicos que regulan esta activación; son críticos para mantener la autotolerancia y modular la duración y amplitud de la respuesta inmune. Sin embargo, son también los principales mecanismos que el melanoma explota para mantener su crecimiento evadiendo al sistema inmune. El primer "checkpoint" es la interacción entre CPA y LT, mediante proteínas que inactivan a LT, siendo la más importante CTLA-4.

El ipilimumab, como se mencionó anteriormente, es un anticuerpo monoclonal que se une a CTLA-4, bloqueando la inhibición de éste a la activación, proliferación y efecto de los linfocitos T. Esto disminuye la inmunotolerancia y puede promover la regresión tumoral.

Esta droga ha demostrado un beneficio clínico en 2 estudios prospectivos randomizados ${ }^{2,3}$. 
Hodi et al. ${ }^{2}$, aleatorizó 676 pacientes con melanoma metastásico previamente tratados con alguna terapia sistémica, a recibir Ipilimumab más una vacuna gp100, ipilimumab solo o la vacuna gp100 sola. La mediana de sobrevida global para el grupo de ipilimumab solo fue de 10 meses, la de la rama de ipilimumab más la vacuna fue de 10,1 meses, comparado con 6,4 meses para el grupo que recibió la vacuna sola. El análisis luego de 1 año mostró que $44 \%$ de los pacientes tratados con ipilimumab y $45 \%$ de los pacientes tratados con ipilimumab más la vacuna gp100 estaban vivos, comparados con $25 \%$ de los pacientes tratados solamente con la vacuna gp100. En cuanto a los efectos adversos, este estudio reportó 10 a $15 \%$ de eventos adversos inmuno relacionados (EAir) grado 3 o 4, incluyendo principalmente diarrea o colitis, y otros eventos endocrinológicos como inflamación pituitaria. Estos efectos cedieron al suspender la droga y administrar corticoides, y en 4 casos requirió el uso de infliximab, un anticuerpo monoclonal contra el factor de necrosis tumoral. Hubo 14 muertes relacionadas al uso del fármaco $(2,1 \%)$.

Por su parte, Robert et al. ${ }^{3}$, aleatorizó 502 pacientes con melanoma metastásico que no habían recibido terapias sistémicas previamente, en 2 ramas: una recibió ipilimumab más dacarbazina y la otra placebo más dacarbazina. La mediana de sobrevida global para el grupo con ipilimumab fue de 11,2 meses, comparado con 9,1 meses en el grupo placebo. La tasa de sobrevida estimada a 1, 2 y 3 años para el grupo ipilimumab fue de $47,3 \%, 28,5 \%$ y $20,8 \%$. Para el grupo placebo fue de $36,3 \%, 17,9 \%$ y $12,2 \%$. En cuanto a los efectos adversos, este estudio reportó $38,1 \%$ de eventos adversos inmuno relacionados (EAir) grado 3 o 4, los más comunes fueron la enterocolitis y hepatitis. No se reportó muertes asociadas al uso de la droga.

Estos son los primeros estudios que demuestran que una droga es capaz de mejorar la sobrevida global en pacientes con melanoma. Si bien los resultados en sobrevida son discretos, aquellos pacientes que responden a tratamiento (15-20\%) son capaces de lograr una prolongada sobrevida libre de progresión, alcanzando un plateau desde el tercer año y con pacientes que llevan vivos sin enfermedad por cerca de una década. Parte de la investigación actual está enfocada en encontrar cuáles son los pacientes que se benefician de este tipo de terapias y así evitar toxicidad innecesaria.

\section{Anti PD-1 y PD-L1: Pembrolizumab-Nivolumab}

Un segundo "checkpoint" es PD-1 ("Programmed Death-1"), una proteína expresada por los linfocitos $\mathrm{T}$ activados. Igual que CTLA-4, regula la activación del linfocito y con ello la tolerancia inmunológica, pero esto ocurre a nivel periférico en la interacción entre el LT y la célula tumoral. Requiere de su ligando, PD-L1, para limitar la respuesta de la célula $\mathrm{T}$ en la periferia, y el daño citotóxico. PD-L1 es expresado en múltiples células y distintos tumores, incluyendo melanoma, impidiendo una respuesta inmunológica efectiva. Tanto pembrolizumab como nivolumab son anticuerpos monoclonales humanizados dirigidos contra PD-1. Tras demostrar tasas de respuesta sobre $25 \%$ y medianas de sobrevida mayores a 16 meses, ambas lograron aprobación acelerada por la FDA.

El pembrozilumab es un anticuerpo monoclonal que se une al receptor PD-1, evitando así la unión con sus ligandos PD-L1 y PD-L2. La vía PD-1 actúa como mediador del agotamiento de las células $\mathrm{T}$. El bloqueo de esta vía lleva a una activación de las células T. Recientemente, en septiembre de 2014, la FDA extendió una aprobación acelerada para el pembrozilumab para pacientes con melanoma metastásico, que han progresado luego del uso de ipilimumab o vemurafenib. Esta aprobación acelerada se concedió luego de conocer los resultados de un estudio randomizado y controlado ${ }^{7}$. En este estudio, Robert et al., aleatorizó 173 pacientes con melanoma metastásico o irresecable, que sufrieron progresión de la enfermedad dentro de las 24 semanas posteriores a la última dosis de ipilimumab o vemurafenib, a recibir pembrolizumab $2 \mathrm{mg} / \mathrm{kg}$ cada tres semanas en una rama, o $10 \mathrm{mg} / \mathrm{kg}$ cada tres semanas en la otra rama. El outcome primario fue la tasa de respuesta global (TRG) de acuerdo a la versión 1,1 de RECIST (Response Evaluation Criteria In Solid Tumor). La TRG fue de $26 \%$ en ambos grupos. La FDA, por lo tanto, aprobó la dosis de $2 \mathrm{mg} / \mathrm{kg}$. Actualmente existe evidencia demostrando beneficio en sobrevida comparado con quimioterapia tanto en primera como en segunda línea para ambas drogas.

Si bien también se describen fenómenos autoinmunes, son menos frecuentes que con Ipilimumab, a excepción de la neumonitis autoinmune, siendo este evento más frecuente cuando se utiliza en pacientes con cáncer pulmonar. Algunos estudios han sugerido que la presencia de PD-L1 en el tumor $o$ en su microambiente podría ser un biomarcador predictor de respuesta; esto no ha sido validado prospectivamente.

Como estos dos tipos de inmunoterapia (anti CTLA4 y anti PD-1) tienen distinto mecanismo de acción, tiene lógica estudiar su potencial sinergismo. Un pequeño estudio mostró que la combinación fue capaz de alcanzar tasas de respuesta de $42 \%$ (17\% de respuesta completas, $25 \%$ respuestas parciales) con una tasa de eventos adversos inmunológicos similar a lo reportado con monoterapia ${ }^{8}$. Una actualización reciente confirmó que estas respuestas 
son prolongadas y que la mediana de sobrevida casi alcanzó los 40 meses $^{9}$ con una sobrevida a 1 año de $94 \%$ y a 2 años de $88 \%$. Este esquema está siendo evaluado en un estudio Fase III aún no publicado al momento de finalizar esta revisión.

\section{Terapias Target}

\section{Inhibidores de BRAF: Vemurafenib}

Aproximadamente, el $50 \%$ de los melanomas cutáneos expresan una mutación activante de BRAF. Al estar activada esta oncoproteína es capaz de estimular la proliferación celular e inhibir la apoptosis. Vemurafenib es un inhibidor específico de la señalización intracelular producida por el BRAF mutado. Dos estudios aleatorizados demostraron la efectividad de esta terapia, lo que condujo a su aprobación por la FDA en el 2011.

Chapman et al. ${ }^{10}$, aleatorizó 675 pacientes con melanoma metastásico o irresecable que expresaban la mutación BRAF V600 (la mutación más frecuente) a recibir vemurafenib o dacarbazina. Al realizar el análisis interino planeado, se concluyó que tanto la sobrevida global como la sobrevida libre de progresión habían alcanzado los criterios pre establecidos para significancia estadística a favor de vemurafenib, y recomendó que los pacientes en la rama de dacarbazina se les autorizara recibir vemurafenib. En cuanto a los efectos adversos, $38 \%$ de los pacientes requirieron una modificación de la dosis o interrupción del tratamiento. Los efectos adversos más frecuentes fueron la aparición de carcinoma espinocelulares, queratoacantomas, fotosensibilidad, artralgias y fatigas.

Por su parte, Sosman et al. ${ }^{11}$, realizó un estudio multicéntrico, fase II, en el que enroló a 132 pacientes con melanoma metastásico con mutación de BRAF V600. Un comité independiente de revisión reportó una tasa de respuesta de $53 \%$, con 8 pacientes logrando una respuesta completa $(6 \%)$. La mediana de duración de la respuesta fue de 6,7 meses.

\section{Inhibidores de BRAF: Dabrafenib}

Es un inhibidor selectivo de BRAF, aprobado por la FDA en el 2013 para pacientes con melanoma metastásico con test positivo para mutación de BRAFV600E.

Hauschild et al. ${ }^{12}$, aleatorizó 250 pacientes con melanoma metastásico o irresecable con mutación de BRAFV600E a recibir dabrafenib o dacarbazina. La mediana de sobrevida libre de progresión fue de 5,1 meses para dabrafenib comparado con 2,7 meses para dacarbazina. Los efectos adversos más frecuentes fueron hiperqueratosis, queratosis verrucosas, queratodermia plantar, fiebre, fatiga, cefalea y artralgia. Se desarrollaron carcinoma espinocelulares o queratoacantomas en 12 pacientes, carcinoma basocelulares en 4 pacientes, micosis fungoide en 1 paciente, y un nuevo melanoma en 2 pacientes.

\section{Inhibidores de MEK: Trametinib}

Los inhibidores de MEK (mitogen-activated ERK-[extracellular signal-regulated kinase] activating kinase) son moléculas pequeñas que inhiben selectivamente a MEK1 y MEK2. BRAF activa MEK1 y MEK2, las que a su vez activan aguas abajo al resto de la vía de la MAP kinasas. Los estudios pre clínicos sugieren que trametinib podría inducir muerte celular en aquellos melanomas que expresen mutaciones de BRAF.

La FDA, en el año 2013 aprobó el uso de trametinib para pacientes con melanoma metastásico o irresecable con mutación de BRAF V600E o K.

La principal evidencia que avala el uso de trametinib fue publicada por Flaherty et al. ${ }^{13}$, en el estudio METRIC, quien aleatorizó 322 pacientes (se permitió ingresar pacientes tratados previamente pero no con inhibidores de BRAF o MEK) a recibir trametinib o quimioterapia intravenosa (dacarbazina o paclitaxel). La sobrevida libre de progresión fue de 4,8 meses en los pacientes tratados con trametinib, comparado con 1,5 meses en el grupo tratado con quimioterapia. Se documento un $35 \%$ de eventos adversos que llevaron interrupción de tratamiento con trametinib, y $27 \%$ de eventos que requirieron de una reducción de dosis. Los eventos adversos más comunes fueron rash cutáneo (erupción acneiforme), diarrea, náuseas, vómitos, fatiga, edema periférico, alopecia, hipertensión y constipación. También se describieron eventos adversos poco frecuentes pero graves como cardiomiopatías, enfermedad pulmonar intersticial, retinopatía y oclusión venosa retinal.

\section{Terapias combinadas}

\section{Dabrafenib combinado con Trametinib}

La resistencia a los inhibidores de BRAF se ha asociado a la reactivación de otras vías, por ejemplo MAPK, por lo tanto, la combinación de inhibidores de transducción que bloqueen una misma vía en distintos sitios puede resultar en una disminución de la resistencia y por lo tanto, un mejor resultado. Un pequeño pero importante trabajo Fase II $^{14}$ aleatorizó 162 pacientes con melanoma irresecable o metastásico a recibir dabrafenib solo o en combinación con trametinib. La tasa de respuesta en el grupo que utilizó la combinación fue de $76 \%$, con una duración promedio de 10,5 meses, comparado con una tasa de respuesta de 54\% y duración promedio de 5,6 meses en el grupo que utilizó dabrafenib solo. También 
hubo una disminución en el desarrollo de carcinoma espinoceluar cutáneo (19\% en el grupo dabrafenib y $7 \%$ en el grupo dabrafenib + trametinib). La aprobación completa de esta terapia combinada va a depender de los resultados que logren demostrar una mejoría en sobrevida libre de progresión.

\section{Discusión}

El desarrollo de nuevas inmunoterapias y terapias target ha cambiado significativamente la forma en que estamos tratando a nuestros pacientes con melanoma metastásico, irresecable o recurrente. Desde el año 2011, 5 drogas fueron aprobadas para su uso en enfermedad avanzada y es probable que les sigan otras.

El aporte más importante de estas nuevas terapias, aparte de las respuestas globales, que en algunos casos pueden ser consideradas bastante modestas (con un grupo creciente de pacientes con buenas y prolongadas respuestas), es definir una línea de investigación para el desarrollo de nuevas terapias, y sobre todo, mejorar las terapias ya existentes, buscando por ejemplo, los mecanismos de resistencia primarios y de resistencia adquirida. Los más optimistas sostienen que probablemente estos pacientes actualmente considerados fuera del alcance terapéutico, podrán tener sobrevidas prolongadas, e incluso considerar una cura para el melanoma metastásico $^{15}$.

Es necesario eso sí, ser muy prudentes en la aplicación de estas nuevas terapias, y conocer muy bien sus limitaciones. En el caso del ipilimumab, se debe tener especial precaución con la toxicidad autoinmune, además, debemos tener en cuenta que muchas veces, la respuesta clínica puede demorar meses en manifestarse, y en ocasiones puede existir un crecimiento inicial antes de objetivarse respuesta. Sin embargo, cuando se produce una respuesta clínica adecuada, por lo general suele ser bastante duradera.

Por su parte, los inhibidores de BRAF tienen mejores tasas de respuesta en torno al $50 \%$ en pacientes con mutación V600 del gen BRAF, y su respuesta clínica se puede ver luego de unos pocos días o semanas de iniciado el tratamiento, pero lamentablemente, la mediana de duración es de sólo 5 a 6 meses.

Es muy importante tener en cuenta que se está generando un nuevo grupo de pacientes con indicaciones quirúrgicas que antes no existían. Se ha observado, sobre todo con el uso de inmunoterapia, grupos de pacientes con enfermedad metastásica con respuestas completas en muchos sitios, pero que no logran controlar una metástasis específica, en los que se hace necesario una resección quirúrgica de ésta.
También se ha observado reducción en el tamaño de lesiones inoperables, generalmente luego del uso de vemurafenib, que luego se pueden resecar quirúrgicamente. Sin duda que se abre un nuevo paradigma en el tratamiento quirúrgico de los pacientes en etapa III irresecable o etapa IV luego del uso de estas nuevas terapias sistémicas.

Es bueno ver, luego de tanto tiempo sin muchos avances concretos para este tipo de pacientes, avances concretos y novedosos, los que nos debería no sólo poner contentos, sino que esperanzados y optimistas en una nueva modalidad de tratamiento para nuestros pacientes oncológicos, en aquellos casos en que la cirugía no es suficiente para tratar una enfermedad.

\section{Referencias}

1. Siegel R, Miller K, Jemal A. Cancer statistics, 2015. CA Cancer J Clin 2015;65:5-29.

2. Hodi FS, O'Day SJ, McDermott DF, Weber RW, Sosman JA, Haanen JB, et al. Improved survival with ipilimumab in patients with metastatic melanoma. N Engl J Med. 2010;363:711-23.

3. Robert C, Thomas L, Bondarenko I, O'Day S, Weber $\mathrm{J}$, Garbe, et al. Ipilimumab plus dacarbazine for previously untreated metastatic melanoma. N Engl J Med. 2011;364:2517-26.

4. Topalian SL, Hodi FS, Brahmer JR, Gettinger SN, Smith DC, McDermott DF, et al. Safety, activity, and immune correlates of anti-PD-1 antibody in cancer. N Engl J Med. 2012;366:2443-54.

5. Hamid O, Robert C, Daud A, Hodi FS, Hwu WJ, Kefford R, et al. Safety and tumor responses with lambrolizumab (anti-PD-1) in melanoma. N Engl J Med. 2013;369:134-44.

6. Davies H, Bignell GR, Cox C, Stephens P, Edkins S, Clegg S, et al. Mutations of the BRAF gene in human cancer. Nature 2002;417(6892):949-54.

7. Robert C, Ribas A, Wolchok JD, Hodi FS, Hamid O, Kefford R, et al. Anti-programmed-death-receptor-1 treatment with pembrolizumab in ipilimumab-refractory advanced melanoma: a randomised dose-comparison cohort of a phase 1 trial. Lancet 2014;384(9948):110917.

8. Wolchok JD, Kluger H, Callahan MK, Postow MA, Rizvi NA, Lesokhin AM, et al. Nivolumab plus ipilimumab in advanced melanoma. N Engl J Med. 2013;369:12233.

9. Sznol M, Kluger HM, Callahan MK, Postow MA, Gordon RA, Segal NH, et al. Survival, response duration, and activity by BRAF mutation status of nivolumab (NIVO, anti-PD-1, BMS-936558, ONO-4538) and ipilimumab concurrent therapy in advanced melanoma (abstract LBA9003). 2014 American Society of Clinical Oncology (ASCO) meeting. 
10. Chapman PB, Hauschild A, Robert C, Haanen JB, Ascierto P, Larkin J, et al. Improved survival with vemurafenib in melanoma with BRAF V600E mutation. N Engl J Med. 2011:364:2507-16.

11. Sosman JA, Kim KB, Schuchter L, González R, Pavlick AC, Weber JS, et al. Survival in BRAF V600-mutant advanced melanoma treated with vemurafenib. N Engl J Med. 2012;366:707-14.

12. Hauschild A, Grob JJ, Demidov LV, Jouary T, Gutzmer R, Millward M, et al. Dabrafenib in BRAFmutated metastatic melanoma: a multicentre, openlabel, phase 3 randomised controlled trial. Lancet 2012;380(9839):358-65.
13. Flaherty KT, Robert C, Hersey P, Nathan P, Garbe C, Milhem $\mathrm{M}$, et al. Improved survival with MEK inhibition in BRAF-mutated melanoma. N Engl J Med. 2012;367:107-14.

14. Flaherty KT, Infante JR, Daud A, González R, Kefford RF, Sosman J, et al. Combined BRAF and MEK inhibition in melanoma with BRAF V600 mutations. N Engl J Med. 2012;367:1694-703.

15. Prieto PA, Yang JC, Sherry RM, Hughes MS, Kammula US, White DE, et al. CTLA-4 blockade with ipilimumab: Long-term follow-up of 177 patients with metastatic melanoma. Clin Cancer Res. 2012;18:203947. 